\title{
Linx
}

Revue des linguistes de l'université Paris X Nanterre

$77 \mid 2018$

Regards croisés sur le futur en français et dans différentes langues romanes

\section{Le futur dans les concessives en espagnol}

\section{Carmen Ballestero de Celis}

\section{(2) OpenEdition}

1 Journals

Édition électronique

URL : http://journals.openedition.org/linx/2798

DOI : $10.4000 /$ linx.2798

ISSN : 2118-9692

Éditeur

Presses universitaires de Paris Nanterre

\section{Édition imprimée}

Date de publication : 30 octobre 2018

Pagination : 143-158

ISBN : 978-2-84016-329-9

ISSN : 0246-8743

Référence électronique

Carmen Ballestero de Celis, «Le futur dans les concessives en espagnol », Linx [En ligne], 77 | 2018, mis en ligne le 30 avril 2019, consulté le 03 mai 2019. URL : http://journals.openedition.org/linx/2798 DOI : 10.4000/linx.2798

Ce document a été généré automatiquement le 3 mai 2019.

Département de Sciences du langage, Université Paris Ouest 


\title{
Le futur dans les concessives en espagnol
}

\author{
Carmen Ballestero de Celis
}

\section{Introduction}

1 La réflexion à l'origine de ces pages naît d'une observation faite par les académiciens dans la dernière grammaire de la Real Academia Española, la Nueva Gramática de la Lengua Española (2009 : 3607) à propos de l'emploi du futur dans les concessives. Dans le chapitre consacré à ces constructions les académiciens affirment :

2 En general, las prótasis concesivas presentan menos restricciones temporales que las condicionales. Así, no rechazan el presente o el pretérito perfecto compuesto de subjuntivo, lo que da lugar a contrastes como aunque lo \{vea haya visto\} $\sim{ }^{*}$ si lo \{vea haya visto\}. Tampoco se extiende a ellas el rechazo del futuro (aunque lo verá $\sim$ * si lo verá) o el del condicional (aunque lo haría *si lo haría).

3 [En général, les protases concessives présentent moins de restrictions temporelles que les conditionnelles. Par exemple, elles ne refusent pas le présent ou le passé du subjonctif, ce qui donne lieu à des contrastes comme aunque lo \{vea haya visto $\sim *$ si lo \{vea haya visto\}. Elles ne sont pas non plus concernées par le refus du futur (aunque lo verá $\sim$ * si lo verá) ou du conditionnel (aunque lo haría $\sim$ *si lo haría).]

4 L'objectif affiché de ces lignes est d'essayer d'expliquer pourquoi, à la différence des conditionnelles, les concessives tolèrent les formes du futur et pourquoi cet emploi reste néanmoins un emploi rare en espagnol moderne. Pour cela il semble nécessaire de proposer une explication intégrée de l'emploi de ce temps verbal, autrement dit, une explication qui tienne compte du fait qu'il fait partie d'un sous-ensemble de formes avec lesquelles il partage des traits formels bien distincts. 


\section{Les propositions explicatives de la grammaire traditionnelle}

5 Il est primordial de s'intéresser aux propositions explicatives que la grammaire traditionnelle fournit pour rendre compte de la forme verbale employée dans une concessive, car la sélection modale qui y est opérée est sans doute l'une des questions les plus controversées qui se pose en linguistique appliquée à l'enseignement des langues. Différentes sont les oppositions auxquelles on a recours pour expliquer le mode employé dans ce type de séquences. La plus répandue est celle qui consiste à expliquer le mode indicatif comme le mode de la réalité et le subjonctif comme le mode de la non-réalité, démarche qui se fonde sur la certitude que le mode d'une forme verbale est déterminé par l'ancrage ou le non-ancrage référentiel de l'opération exprimée par une forme verbale. C'est l'explication proposée par la Real Academia española en 1973 (557-558) :

Las subordinadas concesivas pueden hallarse en indicativo o en subjuntivo. En el primer caso se afirma la existencia efectiva de un obstáculo para el cumplimiento de lo enunciado en la principal; pero la dificultad se rechaza por ineficaz: en Aunque llueve, saldré, la lluvia es un hecho real. Cuando el verbo subordinado está en subjuntivo, la dificultad se siente como posible: Aunque llueva, saldré, la lluvia es una dificultad posible.

[On peut trouver des subordonnées concessives à l'indicatif ou au subjonctif. Dans le premier cas, on affirme l'existence effective d'un obstacle pour l'accomplissement ce qui est énoncé dans la principale; mais cette difficulté est écartée parce que inefficace: dans Aunque llueve, saldré, la pluie est un fait réel. Lorsque le verbe conjugué est au subjonctif, la difficulté est perçue comme possible : Aunque llueva, saldré, la pluie est une difficulté possible.]

6 Cette explication suscite néanmoins deux objections majeures. D'une part, le subjonctif n'est pas seulement le mode de l'hypothétique, comme l'expliquait déjà José Vallejo en 1922, llueva, la forma subjonctive, peut faire référence aussi bien à une pluie éventuelle qu'à une pluie réelle. En voici un exemple :

(1) Media hora después de la llegada del cadáver a Ermua empezó a llover, sin que nadie se moviera de su sitio frente al Ayuntamiento. "No nos vamos aunque llueva", añadieron los manifestantes en sus consignas, que llegaron incluso a decir: "No es agua, es su sangre", entre gritos de "ETA, asesina" y "No estamos todos, falta Miguel". (ABC Electrónico, 14/07/1997)

[Une demi-heure après l'arrivée du cadavre à Ermua il a commencé à pleuvoir, sans que personne ne bouge de sa place face la Mairie. « Nous ne partons pas, même s'il pleut ", ont ajouté les manifestants dans leurs consignes, où l'on pouvait même entendre : «Ce n'est pas de l'eau, c'est du sang », entre les cris de «ETA, assassins » et « Nous ne sommes pas tous là, il manque Miguel ».]

D'autre part il est difficile de soutenir qu'une concessive à l'indicatif affirme l'existence effective du fait auquel il est fait référence si l'on tient compte du fait que le conditionnel était déjà considéré comme un temps de l'indicatif.

Conscients de l'inadéquation de la proposition académique, Alexander Veiga et Mosteiro Mosteiro Louzao ( 2006 : 116) font remarquer que, malgré la révision que Margarita $\mathrm{H}$. Van Rens (1977:11-17) réalisa des grammaires académiques, l'explication des modes verbaux dans les propositions dites concessives ne fut pas modifiée :

Las gramáticas académicas de 1931 y 1973, junto con la transformativa de Hadlich (1971), serían objeto de revisión en cuanto a ciertas afirmaciones sobre la sintaxis 
de las oraciones concesivas por parte de Van Rens (1977), si bien dicha revisión dejó al margen el tema de los modos verbales.

[Les grammaires de l'Académie de 1931 et 1973, et celle transformationnelle de Hadlich (1971), furent révisées par Van Rens (1977) en ce qui concerne certaines affirmations sur la syntaxe des propositions concessives, mais cette révision n'a pas tenu compte des modes verbaux.]

Ce qui nous intéresse ici c'est le fait que l'on ne retrouve aucun exemple avec une forme de futur dans les lignes qui suivent, sans doute parce qu'il est difficile de soutenir qu'un événement qui n'a pas encore eu lieu soit ancré dans la réalité extralinguistique. Parmi les grammaires espagnoles écrites en français qui adoptent cette explication (la Grammaire de l'espagnol moderne de Jean-Marc Bedel, la Grammaire espagnole de Jean Bouzet, la Syntaxe de l'espagnol moderne de Jean Coste et Augustin Redondo, etc.), aucune ne fournit non plus d'exemples avec des formes de futur. Pour illustrer des concessives portant sur des faits réels, ces grammaires se contentent des formes du présent et du passé, ce qui incite à croire que le futur ne peut pas avoir de valeur factuelle.

Une application différente de cette opposition réalité / non-réalité est celle proposée dans la Gramática de la lengua española écrite par Emilio Alarcos Llorac (1994 : 153) :

1) Aunque ganas, ese negocio no es bueno.

Aunque ganabas, ese negocio no era bueno.

Aunque ganaste, ese negocio no fue (era) bueno.

2) Aunque ganarás, ese negocio no es (será, era, sería) bueno.

Aunque ganarías, ese negocio no es (será) bueno.

3) Aunque ganes, ese negocio no es (será) bueno.

Aunque ganases (ganaras), ese negocio no es (será, era...) bueno.

Aunque ganares, ese negocio no será bueno.

[...] se observa que en la serie 1) la ganancia a que se alude se considera real o efectiva; que en la serie 2) esa ganancia se estima como posible (o sea, se ignora pero no se excluye la eventualidad de la ganancia), finalmente, en la serie 3) la ganancia se manifiesta como ficticia (es decir que el hablante cree que lo real es que no se gana). Este triple enfoque depende exclusivamente de cómo considera el hablante los hechos, esto es, de su actitud al evaluar el grado de realidad que atribuye a los hechos denotados.

[(...) on observe que dans la série 1) les gains auxquels il est fait référence sont considérés comme étant réels ou effectifs ; dans la série 2) ces gains sont estimés comme possibles (c'est-à-dire, on ignore mais on n'exclut pas l'éventualité des gains), enfin, dans la série 3) les gains apparaissent comme fictifs (c'est-à-dire, le sujet parlant croit que la réalité est que l'on ne gagne pas). Cette triple approche dépend exclusivement de la manière dont le sujet parlant considère les faits, autrement dit, de son attitude au moment d'évaluer le degré de réalité qu'il attribue aux faits dénotés.]

11 Selon E. Alarcos, l'emploi du futur ne répond plus à l'expression d'un fait dont la réalisation est effective mais bien au contraire, elle serait contingente, dans les mots de l'auteur : «La de los hechos cuya realidad es factible siempre que se cumplan ciertas condiciones (el paso del tiempo, el cambio de circunstancias u otros factores)» (1994: 153-154). En d'autres termes, le futur serait le temps du possible.

L'explication proposée par Alarcos distingue ainsi trois cas de figure selon le caractère réel, possible ou fictif de l'événement exprimé par la concessive. Cela implique au moins deux affirmations difficiles à soutenir : d'une part que le futur dit la même contingence que le conditionnel (il exprimerait, comme le conditionnel, ce qui est possible), d'autre part, que les formes subjonctives n'expriment que des évènements fictifs, plus concrètement, qu'elles ne font référence qu'à des événements contrefactuels. Or aunque 
ganes et aunque ganases (ganaras) peuvent non seulement faire référence à des événements réalisables, mais ces séquences peuvent aussi référer à des événements réalisés ou qui sont en train de se réaliser, comme dans les exemples suivants :

(2) Cuando tenía el programa de televisión recibía muchos comentarios sobre el título. Me decían que si me refería a que los escritores son pretenciosos o vanidosos. Yo les decía que la literatura es un vano oficio porque, aunque ganes el Nobel o solo vendas cien ejemplares de un libro, al final de la vida no te queda nada. (Jarque, Fietta: «El fracaso es un aprendizaje». El País.com. Babelia. Madrid: elpais.com/ suple/babelia, 2011-06-11.)

[Lorsque j'avais l'émission de télévision je recevais beaucoup des commentaires sur le titre. On me demandait si je faisais référence au fait que les écrivains sont prétentieux ou vaniteux. Je leur disais que l'écriture est un métier vain parce que, même si tu gagnes le Nobel ou que tu vends seulement cinq exemplaires d'un livre, à la fin de ta vie il ne te reste rien.]

(3) Ya es hora de que la Administración sea condenada a pagar las costas, hasta ahora, aunque ganaras tenías que soportar los costes del abogado.

(http://www.elperiodicodearagon.com/noticias/aragon/condenada-dga-imponermulta-45-000-sin-base_854249.html)

[Il est temps que l'Administration soit condamnée à payer les frais de justice, jusqu'à maintenant, même si tu gagnais tu devais supporter les frais d'avocat.]

Une autre opposition à laquelle a eu recours la grammaire traditionnelle pour expliquer la forme verbale d'une concessive est celle qui oppose l'indicatif comme le mode de l'expérience au subjonctif comme le mode de la non-expérience. James R. Shawl fut l'un des premiers à avoir recours à cette opposition. Dans une étude consacrée au subjonctif espagnol, "Syntactic Aspects of the Spanish Subjunctive » (1975:323-329), il présente, dans une perspective générativiste, une analyse syntaxique de diverses propositions où les deux modes sont possibles. Pour expliquer cette alternance il fait appel à cette opposition expérience/non-expérience. À l'instar de J. R. Shawl, certains auteurs considèrent que l'indicatif est le mode de ce qui peut être vérifié empiriquement et le subjonctif le mode de ce qui ne peut pas être prouvé en s'appuyant seulement sur l'expérience. C'est l'explication proposée par Jesús Fernández Álvarez dans son ouvrage $E l$ subjuntivo (1987 : 59). L'auteur y explique la sélection modale opérée dans la proposition introduite par aunque à travers la même opposition expérience / non-expérience :

Esto quiere decir que el indicativo (experiencia) se emplea cuando el hablante ha experimentado o conoce con certeza el hecho introducido por aunque; mientras que el subjuntivo (no experiencia) se usa cuando el hablante no tiene experiencia personal de la acción, o aunque la tenga, le interesa, le conviene o simplemente prefiere presentarla como dudosa y, en su consecuencia, como no experimentada.

[Ceci veut dire que l'indicatif (expérience) est employé lorsque le sujet parlant a expérimenté ou connaît avec certitude le fait introduit par aunque; alors que le subjonctif (non expérience) est utilisé lorsque le sujet parlant n'a pas d'expérience personnelle de l'action, ou même s'il l'a, il tient à la présenter, il a intérêt ou simplement il préfère la présenter comme douteuse et, par conséquent, comme pas expérimentée.]

Dans la tradition grammaticale française, cette même explication est proposée par Pierre Gerboin et Christine Leroy dans leur Grammaire d'usage de l'espagnol contemporain (1994: 489). À la question "Comment choisir le mode de la subordonnée de concession", les auteurs répondent :

Le choix principal, pour le mode de la subordonnée de concession, se fait entre l'indicatif et le subjonctif. C'est la valeur de ces modes (voir 233), qui justifie en espagnol leur emploi : 
- si la concession porte sur un fait réalisé ou considéré comme réalisé par le locuteur, c'est le mode indicatif qui est utilisé

- si la concession porte sur un fait qui n'est pas - ou pas encore - réalisé ou considéré comme tel par le locuteur, c'est le mode subjonctif qui est utilisé.

D'après ces lignes, toute concessive faisant référence à un fait présent ou passé se construit à l'indicatif et toute concessive référant à un fait futur se construit au subjonctif. Cette explication ne permet pas de rendre compte des constructions qui tout en faisant référence à un fait réalisé, se construisent au subjonctif. Et elle ne permet pas non plus, et c'est justement ce qui nous intéresse dans ces pages, d'expliquer toutes les propositions introduites par aunque qui, portant sur un fait qui n'est pas encore réalisé, se construisent avec des formes verbales de futur, un temps que la tradition, aussi bien espagnole que française, inclut dans le mode indicatif. Un énoncé comme le suivant resterait inexpliqué :

(4) Aunque lo olvidaré, aún no lo olvido, y cuando ahora miro el retrato pequeño de mi imposible tía Teresa que Ranz conserva en su casa, lo miro con más atención de la que le presté jamás, durante mi infancia y mi adolescencia. (J. Marías, Corazón tan blanco, p. 297)

[Même si je vais l'oublier, je ne l'oublie pas encore, et maintenant, lorsque je regarde le petit portrait de mon impossible tante Teresa que Ranz garde chez lui, je le regarde avec plus d'attention que je ne lui ai jamais prêtée, pendant mon enfance et mon adolescence.]

16 Cet énoncé resterait inexpliqué car, dans la mesure où la concession porte sur un fait qui n'est pas - ou pas encore - réalisé, lo olvidaré, il aurait fallu utiliser, selon P. Gerboin et C. Leroy, une forme subjonctive, il aurait fallu dire Aunque lo olvide. Autrement dit, pour ces auteurs l'emploi du futur est banni de la proposition concessive.

\section{Le regard de la linguistique du signifiant}

17 Un regard différent, celui de la linguistique du signifiant, permet non seulement de rendre compte de façon plus objective et rigoureuse de la sélection modale qui y est opérée, cette approche permet aussi d'expliquer la rareté de certains emplois. Si l'on s'attarde sur la matérialité du futur, il est facile de constater que, comme le conditionnel, il présente dans toutes les formes de son paradigme un « $\mathrm{r}$ », morphème dont la valeur n'a cessé de faire débat. Or, comme l'argumente Sophie Azzopardi dans son travail de thèse (2011: 103-104), tout pousse à penser que c'est la valeur temporelle qui définit ce morphème :

la valeur temporelle d'ultériorité était déjà présente dans la valeur de virtualité de l'infinitif, et [...] c'est par effacement de ce signifié premier, toujours présent dans l'infinitif roman actuel, que la valeur d'ultériorité est devenue le signifié de langue de la structure grammaticalisée.

Pour ce qui est d'une construction concessive, elle se définit, en tant que relation contrastive, par son caractère présuppositionnel : ce qui y est présupposé est une relation implicative selon laquelle un premier terme implique l'absence d'un deuxième, autrement dit, une construction du type $A$ aunque $B$ sous-entend que l'énonciation de B implique l'absence de l'énonciation de A. En d'autres termes Salgo aunque llueve présuppose que la déclaration /llover/ implique l'absence de la déclaration/salir/, car normalement, si nous ne sommes pas des escargots, lorsqu'il pleut on ne sort pas. Voici le schéma illustrant la relation implicative sous-jacente à toute concessive : 
Salgo aunque llueve

A aunque $B$

présuppose $\mathrm{B}>-\mathrm{A}$

19 Ce qui est propre à toute relation contrastive c'est justement la négation de cette implication, de telle sorte que les énonciations de A et de B, la sortie et la pluie, a priori incompatibles, deviennent compatibles.

20 L'énonciation de $B$ se présente alors comme le terme impliquant d'une relation implicative sous-entendue (si j'énonce B je ne devrais pas énoncer A : si j'énonce qu'il pleut je ne devrais pas énoncer que je sors). De ce fait la protase d'une concessive, $B$, se voit située dans une antériorité notionnelle, espace énonciatif opposé à celui instauré par le futur. Tout incite à croire que c'est justement cette discordance temporelle l'ultériorité dite par le futur face à l'antériorité notionnelle dans laquelle se situe la déclaration énoncée dans la concessive - qui explique la rareté de l'emploi des formes verbales du futur dans les protases concessives. Cette même discordance explique la réticence du futur et du conditionnel à apparaître dans les protases conditionnelles. Or, si dans une construction concessive cette discordance se révèle dans ce qui est sousentendu, dans une construction conditionnelle, la discordance est explicitement verbalisée, ce qui explique que, même si l'emploi du futur et du conditionnel n'est pas exclu (dans certaines régions de l'Espagne on peut entendre *Si vendrías), il s'agit d'un emploi qui reste néanmoins minoritaire et est considéré comme non conforme à la norme.

21 Une lecture de la matérialité du paradigme du futur permet aussi de constater l'existence d'un signe spécifique de première personne : cantaré s'oppose à cantará. À la manière de Gilles Luquet, il est considéré ici que le fait que la première personne dispose d'un signifiant propre (ce qui est le cas aussi du présent et du prétérit) est dû à ce que l'opération à laquelle elle renvoie est entendue dans un univers modal directement construit à partir du présent d'actualité, puisque c'est dans le présent que le référent de la première personne s'oppose de manière effective au référent de la troisième personne. L'auteur appelle les formes qui présentent un signe spécifique pour la première personne (le présent, le prétérit et le futur) « formes actualisantes »:

Cantaréłcantará

Canto $\neq$ canta

Canté $\neq$ cantó

22 À l'inverse, le fait que la première personne ne dispose pas d'un signifiant propre est dû à ce que l'opération à laquelle elle renvoie est entendue dans un univers modal qui n'est pas construit, ou pas directement construit, à partir du présent d'actualité, l'univers modal des formes dites "inactualisantes» (l'imparfait, le conditionnel et les formes subjonctives) :

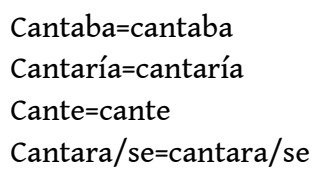

23 Si avec les premières le locuteur asserte l'information qui y est exprimée, en la plaçant dans un premier plan discursif, avec les formes inactualisantes l'information est présentée dans un deuxième plan discursif. Comme le signale G. Luquet, il y a deux raisons qui expliquent qu'un locuteur ne soit pas en mesure d'actualiser un événement : c'est soit parce qu'il ne peut pas le faire, soit parce qu'il ne veut pas le faire. Il ne peut pas le faire lorsque l'évènement exprimé par la forme verbale fait référence à un fait que le 
locuteur considère comme hypothétique ; il décide de ne pas le faire lorsque l'existence de cet événement a déjà été actualisée et constitue de ce fait une information déjà connue de l'interlocuteur. En somme, comme le signale Patricia Lunn (1989:687-702), une information susceptible d'assertion doit présenter deux caractéristiques : être une vérité authentifiée et apporter une information nouvelle. Il y a ainsi deux types d'information que l'on ne peut pas asserter : les informations qui sont inconnues du locuteur et celles qui sont connues de l'interlocuteur, car aucune d'elles ne peut améliorer la représentation du monde de l'interlocuteur.

Si les subjonctifs de (5) et (6) s'expliquent par la même opération d'inactualisation, les raisons discursives qui en sont à l'origine sont différentes :

(5)

Las cosas empiezan y no se sabe dónde pueden acabar.

El apagón va a dar ánimo a mucha gente que dudaba.

Ciertas cosas sí se sabe cómo van a acabar.

Ojalá te equivoques. Pero aunque tengas razón, Edmundo, hay algo que tú no entiendes: que algunas cosas se hacen porque se tienen que hacer. (Belén Gopegui, Lo real, p. 327)

[Il est des choses qui commencent et on ne sait pas où elles peuvent finir.

La coupure d'électricité va encourager beaucoup de gens qui doutaient.

Certaines choses, on sait bien comment elles vont finir.

J'espère que tu te trompes. Mais même si tu as raison, Edmundo, il y a quelque chose que tu ne comprends pas : il y a des choses que l'on fait parce qu'il faut les faire.]

(6) ¿Entonces para qué necesitas al geriatra?

Para que me recuerde mi edad -dijo Lucas.

No parezco mucho menor que tú, aunque tengas esas arrugas.

No es lo que pareces. Es lo que eres 熙dijo Lucas. (Héctor Aguilar Camín, El error de la

luna, p. 184)

[ $i$ Alors pourquoi tu as besoin du gériatre?

Pour qu'il me rappelle mon âge - dit Lucas.

Je n'ai pas l'air d'être beaucoup plus jeune que toi, même si tu as ces rides.

Ce n'est pas ce à quoi tu ressembles. C'est ce que tu es -dit Lucas.]

En (5) l'interlocuteur d'Edmundo n'est pas sûr qu'Edmundo ait raison justement parce qu'il souhaite qu'il se trompe, et présente donc le fait référé par le subjonctif comme hypothétique. En revanche, en (6) le fait auquel le subjonctif fait référence est présenté par l'interlocuteur de Lucas comme une information partagée : Lucas est censé connaître son aspect physique, davantage s'il envisage d'aller chez le gériatre pour qu'il lui rappelle qu'il est vieux. En (5), on inactualise parce qu'on ne peut pas asserter ce qui est énoncé, en (6) parce que l'on considère qu'il est inutile de l'asserter dans la mesure où il s'agit d'une information déjà actualisée par le contexte.

L'opposition entre un mode actualisant et un mode inactualisant permet non seulement de rendre compte de tous les emplois des formes subjonctives, mais cette opposition permet aussi d'expliquer l'emploi des formes verbales du futur dans une concessive, un emploi rare mais tout à fait en accord avec la norme. Si les formes subjonctives répondent à une opération d'inactualisation, les formes verbales du futur s'expliquent par la capacité et la volonté du locuteur d'actualiser les événements qui y sont exprimés, autrement dit par la capacité et la volonté du locuteur d'asserter le contenu de la concessive. 
Lorsqu'un locuteur souhaite faire porter la concession sur un fait futur, il peut employer aussi bien des formes actualisantes que des formes inactualisantes. Les différentes possibilités sont illustrées dans les exemples qui suivent :

(7) Dr. Soto: Bueno, bueno... Dejemos el tema. Presentas el caso en Oslo... si el resultado es positivo, claro...

Dra. Caruana : Aunque fuera negativo.

Dr. Soto : Ah, ¿sí?

Dra. Caruana: Aunque sea negativo. Tú mismo lo has dicho: no hay constancia de una concepción en coma... con un "score" de cuatro en la escala de Glasgow, lesión supretentorial, arreflexia, rigidez de decorticación...

Dr. Soto : Ya... pero, en cualquier momento, ese embarazo puede...

(Sanchis Sinisterra, José: Sangre lunar. Madrid: Fundamentos, 2010).

[Docteur Soto: Bon, bon...Changeons de sujet. Tu présentes le cas à Oslo...si le résultat est positif, bien sûr...

Docteur Caruana. : Même s'il était négatif.

Dr. Soto : Ah, oui ?

Docteur Caruana : Même s'il est négatif. Tu l'as dit toi-même : on n'a pas constaté une conception dans un état de coma... avec un « score » de quatre selon l'échelle de Glasgow, lésion supratentoriale, aréflexie, rigidité de décortication...

Docteur Soto : Oui...mais, à tout moment cette grossesse, elle peut...]

(8) Nos gustaría hablarles del primer lunes de febrero que tiene muy buena pinta por estos lares dado que aunque sea lunes no sólo es Santa Agueda, también dicha Gadea, sino que hay gran concierto, salvaje, en la sala de la calle Azkuene 17, Mogambo.

(«DONOSTIA, LA TENTACIÓN. Aquellos años, estos conciertos». El Diario Vasco. San Sebastián: Sociedad Vascongada de Publicaciones, 2001-01-31).

[On aimerait vous parler du premier lundi de février qui a l'air très bien dans le coin puisque même si c'est un lundi ce n'est pas seulement la fête de Sainte Agathe, c'est aussi celle de Sainte Gadea, mais il y a un grand concert sauvage, dans la salle de la rue Azkuene 17, Mogambo.]

(9) Por un segundo, Eva la odió. "¿Y ahora?", pensó. Alcanzó a comentar:

Es tarde, sí, aunque mañana es domingo.

(Coscia, Jorge: Juan y Eva. El amor, el odio y la revolución. Buenos Aires: Sudamericana, 2011).

[Pendant une seconde, Eva l'a haï. « Et maintenant ? Elle a réussi à dire :

Il est tard, oui, même si demain c'est dimanche ».]

(10) El humorista Eugenio permanece internado en el hospital madrileño de La Paz aquejado de un proceso cardiovascular agudo, aunque será dado de alta en los próximos días, según informaron fuentes del hospital. (Prensa, El Mundo, $11 / 02 / 1994)$

[L'humoriste Eugenio est toujours admis à l'hôpital madrilène de La Paz souffrant d'un processus cardiovasculaire aigu, quoiqu'il sera autorisé à sortir dans quelques jours, selon des informations émanant de l'hôpital.]

En (7), aunque fuera negativo /aunque sea negativo, le locuteur utilise des formes inactualisantes fuera et sea, non pas parce qu'il ne veut pas asserter le contenu de la concessive, mais parce qu'il ne peut pas le faire, ser negativo étant un fait qui, comme le montre le contexte, relève de l'hypothétique. Le fait que le résultat du test soit négatif n'est ici qu'une éventualité dont le degré de probabilité est marqué par la forme choisie: si le présent de subjonctif dit qu'il s'agit d'un événement possible, un événement qui a des chances de se réaliser (aunque sea negativo = il sera probablement négatif), la forme en -ra, la plus inactualisante du système verbal espagnol, le marque comme improbable (aunque fuera negativo = il sera difficilement négatif). 

en l'occurrence parce qu'il ne veut pas asserter le contenu de la concessive. Il décide de ne pas le faire parce que l'existence de l'événement exprimé par la concessive, ser lunes, est discursivement constatable. L'énoncé commence par dire que l'on parle d'un lundi : Nos gustaría hablarles del primer lunes de febrero ... Dans cet énoncé on aurait pu employer sans problème une forme actualisante, es ou será, mais on aurait l'impression d'une certaine redondance : Nos gustaría hablarles del primer lunes de febrero [...] que aunque es lunes... ou Nos gustaría hablarles del primer lunes de febrero que aunque será lunes... Sea, la forme subjonctive, en tant que forme inactualisante, permet de placer cette information, qui est apparue quelques mots plus haut, dans un deuxième plan discursif.

Or, un locuteur peut aussi faire porter la concession sur un fait futur en utilisant des formes actualisantes. Il décide d'utiliser une forme actualisante lorsque le fait auquel il se réfère dans la concessive est non seulement un fait dont la réalisation est considérée comme acquise, mais aussi dont le locuteur souhaite informer les destinataires, comme en (9). Dans cet énoncé le locuteur décide d'utiliser la forme es parce qu'il s'agit d'un évènement présent qui se prolonge vers le futur. On aurait pu dire également aunque mañana será domingo, avec une forme du futur, mais dans ce cas l'opération verbale serait rattachée uniquement à un moment postérieur au moment de l'énonciation.

31 Enfin, comme en (10), le locuteur peut se servir du futur, une forme verbale également actualisante. S'il se sert du futur c'est parce que le fait auquel il est fait référence dans la concessive est non seulement un fait dont la réalisation est considérée comme acquise dans un moment à venir, mais aussi dont le locuteur souhaite informer les destinataires : la sortie de l'hôpital est présentée comme une information nouvelle pour les lecteurs de El Mundo.

\section{Conclusions}

Une lecture de la matérialité des formes verbales du futur permet dans un premier temps de rendre compte de la rareté de ce temps verbal dans la protase d'une concessive : l'ultériorité dite par le futur est contraire et de ce fait réticente à l'antériorité notionnelle où se situe la déclaration de toute protase concessive. La lecture de son signifiant permet également de rendre compte de son usage: tenir compte de la morphologie de ce temps et l'expliquer comme une forme actualisante permet de soutenir que son emploi est dû à la capacité et à la volonté du locuteur de présenter dans un premier plan discursif un fait dont la réalisation est considérée comme acquise dans un moment à venir, un fait dont l'énonciation était a priori incompatible avec l'énonciation de l'apodose, mais qui finit par constituer le noyau informatif. En effet, il n'est pas surprenant que dans notre corpus, qui comporte deux cent constructions avec une forme du futur, presque quatre-vingt pour cent de ces énoncés soient des occurrences tirées de textes journalistiques, écriture à vocation essentiellement informative. 


\section{BIBLIOGRAPHIE}

ALARCOS LLORACH, E., 1994, Gramática de la lengua española, Espasa-Calpe, Madrid.

AZZOPARDI, S., 2011, « Le Futur et le Conditionnel : valeur en langue et effets de sens en discours. Analyse contrastive espagnol / français », Linguistics, Université Paul Valéry - Montpellier III. French. <tel-00718652>

BEDEL, J. M., 2004 [1997], Grammaire de l'espagnol moderne, Presses Universitaires de France, Paris. BOSQUE, I., 1990, Indicativo y subjuntivo, Taurus Universitaria, Madrid.

BOUZET, J., 2006 [1946], Grammaire espagnole : classes supérieures de l'enseignement secondaire ; préparation à la licence, Belin, Paris.

COSTE, J. et REDONDO, A., 1990 [1965], Syntaxe de l'espagnol moderne, Sedes, Paris.

FERNÁNDEZ ÁLVAREZ, J., 1987, El subjuntivo, Edelsa, Madrid.

FLAMENCO GARCÍA, L., 1999, « Las construcciones concesivas y adversativas », dans V. Demonte et I. Bosque (éds.), Gramática descriptiva de la lengua española, Madrid, Espasa-Calpe, vol. 3, p. 3805-3878.

GERBOIN, P. et LEROY, C., 1994 [1991], Grammaire d'usage de l'espagnol contemporain, Hachette Livre, Paris.

LUNN, P., 1989, « Spanish mood and the Prototype of assertability », Linguistics, 27, p. 687-702.

LUQUET, G., 2004, La teoría de los modos en la descripción del verbo español, Arco/Libros, Madrid.

REAL ACADEMIA ESPAÑOLA, 1973, Esbozo de una nueva gramática de la lengua española, EspasaCalpe, Madrid.

REAL ACADEMIA ESPAÑOLA \& ASOCIACIÓN DE ACADEMIAS DE LA LENGUA ESPAÑOLA, 2009, Nueva gramática de la lengua española, Espasa Libros, Madrid.

RODRÍGUEZ ROSIQUE, S., 2008, Pragmática y Gramática : condicionales concesivas en español, Peter Lang, Frankfurt am Main.

SHAWL, J. R., 1975, « Syntactic Aspects of the Spanish Subjunctive », Hispania, Vol. 58, No. 2, p. 323-329.

VALLEJO, J., 1922, « Notas sobre la expresión concesiva », Revista de Filología Española, n 9, p. 40-51.

VAN RENS, M.H., 1977 « Acerca de la oración concesiva encabezada por aunque », Español actual, nº 32, p. 11-17.

VEIGA RODRÍGUEZ, A. et MOSTEIRO LOUZAO, M., 2006, El modo verbal en cláusulas condicionales, causales, consecutivas, concesivas, finales y adverbiales de lugar, tiempo y modo, Universidad de Salamanca, Salamanca.

Corpus

REAL ACADEMIA ESPAÑOLA, Banco de datos (CREA) [en línea]. Corpus de referencia del español actual. http://www.rae.es/recursos/banco-de-datos/crea 
REAL ACADEMIA ESPAÑOLA, Banco de datos (CORPES) [en línea]. Corpus del español del siglo XXI. http://www.rae.es/recursos/banco-de-datos/corpes-xxi

\section{RÉSUMÉS}

Différentes sont les oppositions auxquelles on a eu recours pour expliquer la forme verbale employée dans une concessive. L'une des plus fréquentes est celle qui oppose l'indicatif comme le mode de l'expérience au subjonctif comme le mode de la non expérience. Dans cette perspective, si l'action de la concessive est réalisée ou considérée comme telle par le locuteur, on doit utiliser l'indicatif, alors que si l'action de la concessive n'est pas - pas encore - réalisée, c'est le subjonctif qui doit y être employé. Or en espagnol une concessive exprimant une action qui n'est pas encore réalisée peut parfaitement se construire avec des formes verbales indicatives, notamment le futur. S'il est vrai, comme le montre notre étude de corpus, que l'emploi de ce temps verbal n'est pas très fréquent dans la protase d'une concessive, son emploi est parfaitement normatif. Le but de cet article est tout d'abord d'expliquer la moindre fréquence des formes du futur dans ce type de séquences pour ensuite proposer une explication intégrée de ce temps verbal. Si l'on s'attarde sur la matérialité de ce temps verbal, tout pousse à penser que c'est sa valeur prospective qui explique la réticence de ce temps à apparaître dans la protase d'une concessive : l'énonciation qui y est exprimée est conçue comme un obstacle a priori inefficace et se situe de ce fait dans une antériorité notionnelle, espace énonciatif opposé à celui instauré par le futur. Une lecture de la matérialité de cette forme verbale permet également d'expliquer la rareté de son emploi dans ce type de séquences: pour l'utiliser il faut que le locuteur ait non seulement la capacité de présenter le fait auquel il est fait référence comme un fait dont la réalisation est acquise dans un moment à venir, mais il faut aussi qu'il ait la volonté d'en constituer le noyau informatif.

The verbal form in concessive constructions is explained by means of different types of oppositions. The most frequent one opposes the indicative as the mode of the experience to the subjunctive as the mode of the non-experience. In this perspective, the indicative mood is used if the action of the concessive clause is realized or considered as such by the speaker, while if the action of the concessive clause is not -not yet-realized, it is the subjunctive which must be used. In Spanish, however, a concessive clause expressing an action which has not been realized yet can perfectly be constructed with indicative verbal forms, in particular the future. If it is true, as shows our corpus study, that the use of this verbal time is not very frequent in the concessive clause, it is nevertheless perfectly normative. The purpose of this article is first to explain the lower frequency of the forms of the future and then to propose an integrated explanation of this tense. If we linger over the materiality of this verbal time, everything leads to think that it is forward-looking value that explains the reluctance of this tense to appear in a concessive clause: the statement expressed is conceived as an obstacle a priori ineffective and it is thus placed in a notional anteriority, an enunciative space opposite to that established by the future. A reading of the materiality of this verbal form also makes it possible to explain the rarity of its use in this type of sequences: to use this tense the speaker must have not only the capacity to present the fact referred to as a fact whose realization is conceived at some point in the future, but also the will to present it as the informative nucleus.

\section{INDEX}

Mots-clés : temps futur, espagnol, propositions concessives

Keywords : future tense, Spanish, concessive clauses 
AUTEUR

CARMEN BALLESTERO DE CELIS

Université de la Sorbonne Nouvelle - Paris 3 / EA 7345 CLESTHIA 